\begin{tabular}{|c|l|}
\hline Title & Generalized Scaling Theory for Critical Phenomena Including Essential Singularities and Infinite Dimensionality \\
\hline Author(s) & Nogawa, Tomoaki; Hasegawa, Takehisa; Nemoto, Koji \\
\hline Citation & $\begin{array}{l}\text { Physical Review Letters, 108(25), 255703 } \\
\text { https://doi.org/L0.1103/PhysRevLett.108.255703 }\end{array}$ \\
\hline Issue Date & 2012-06-22 \\
\hline Doc URL & http://hdl.handle.net/2115/50156 \\
\hline Rights & ○ 2012 A merican Physical Society \\
\hline Type & article \\
\hline File Information & PRL108_25_255703.pdf \\
\hline
\end{tabular}

Instructions for use 


\title{
Generalized Scaling Theory for Critical Phenomena Including Essential Singularities and Infinite Dimensionality
}

\author{
Tomoaki Nogawa* \\ Department of Applied Physics, The University of Tokyo, 7-3-1, Hongo, Bunkyo-ku, Tokyo 113-8656, Japan \\ Takehisa Hasegawa \\ Graduate School of Information Science, Tohoku University, 6-3-09, Aramaki-Aza-Aoba, Sendai, Miyagi 980-8579, Japan \\ Koji Nemoto \\ Department of Physics, Hokkaido University, Kita 10 Nisi 8, Kita-ku Sapporo, Hokkaido 060-0810, Japan
}

(Received 27 January 2012; revised manuscript received 4 April 2012; published 21 June 2012)

\begin{abstract}
We propose a generic scaling theory for critical phenomena that includes power-law and essential singularities in finite and infinite dimensional systems. In addition, we clarify its validity by analyzing the Potts model in a simple hierarchical network, where a saddle-node bifurcation of the renormalizationgroup fixed point governs the essential singularity.
\end{abstract}

DOI: 10.1103/PhysRevLett.108.255703

The scaling theory for the power-law singularity (PLS) of second-order transitions is among the most significant achievements in theoretical physics [1], and provides a comprehensive understanding of critical phenomena with minimal assumptions. The key concept of this theory is an invariance of the singular part of free energy, $g_{d}$, which is scaled by a factor $\hat{b}$ as

$$
g_{d}\left(t, h, L^{-1}\right)=\hat{b}^{-d} g_{d}\left(t \hat{b}^{y_{t}}, h \hat{b}^{y_{h}}, L^{-1} \hat{b}\right) .
$$

Here, $d$ is the dimension of space, $L$ is the linear dimension of the system, and $t$ and $h$ are deviations of model parameters from their critical values (such as reduced temperature $t=T-T_{c}$ and magnetic field $h=H$ ). For the characterization of the criticality, $y_{t}$ and $y_{h}$ are the most fundamental quantities, which lead to critical exponents for the PLS of physical quantities, e.g., $\nu=1 / y_{t}$ and $\beta=\left(d-y_{h}\right) / y_{t}$, corresponding to the correlation length as $\hat{\xi} \propto t^{-\nu}$ and the order parameter as $m \propto t^{\beta}$. The scaling theory is based on the self-similarity owing to the divergence of the correlation length.

There is a kind of continuous transition on which standard scaling theory does not work. The most famous example is the Berezinskii-Kosterlitz-Thouless (BKT) transition in the two-dimensional $X Y$ model [2-4], which exhibits an essential singularity (ES) around the transition point in the disordered phase, where the correlation length diverges as $\hat{\xi} \propto \exp \left[\left(t / t_{0}\right)^{-1 / 2}\right]$ instead of the power law. Below the transition temperature, $\tilde{\xi}$ remains divergent and there appears quasi-long-range order with zero magnetization as if the system stays in criticality. In this sense we hereafter call this phase the critical phase.

A similar singularity has been recently found in infinite dimensional systems. The ES for the infinite dimensional system appears in the ordered phase; the order parameter behaves as $m \propto \exp \left[-\left(t / t_{0}\right)^{-1 / 2}\right]$ toward the transition
PACS numbers: 64.60.ae, 64.60.aq, 75.10.Hk, 89.75.Da

point, above which the critical phase emerges. This is in contrast to the ordinary BKT transition, and is called the inverted BKT singularity [5]. Here we use the words "infinite dimensional" for the property that the typical path length $L$ increases with system volume $N$ as $L \propto$ $\log N$ instead of $N^{1 / d}$. This property is realized in trees, random graphs [6], hierarchical lattices [5], hyperbolic lattices [7], and small-world networks [8] among others. Such systems have been extensively studied in the context of complex networks; the heterogeneous and hierarchical structure has unexpectedly revealed important concepts in physics [9] other than the mean-field behaviors for simpler infinite dimensional systems such as the complete graph. In particular, the ES is found rather often in various models on various infinite dimensional graphs [5,10-17]. Therefore, the ES is considered to be a basic concept of the dynamics in infinite dimensional systems rather than an exotic topic as the BKT transition in finite dimensions. Since inverted BKT singularity is observed in percolation [10-16] and Ising models [5,17], we do not expect its mechanism to be common to that of the ordinary BKT transition, such as vortex-pair condensation [2,3]. Universal understanding of the ES in infinite dimensional systems is still missing.

In this Letter, we propose a generic scaling theory for critical phenomena with ES in infinite dimensional graphs. We also perform renormalization group (RG) analysis of the Potts model in a simple hierarchical network, which confirms the validity of the scaling law.

Now, we consider a scaling formula for infinite dimensional graphs as

$$
g\left(\xi(t)^{-1}, h, N^{-1}\right)=b g\left(\xi(t)^{-1} b, h b^{y_{h}}, N^{-1} b\right) .
$$

Here, $b$ is a scaling factor for the total volume (mass) $N$ and the correlation volume $\xi$ of the system. The lengthbased expression is obtained by replacing $N \rightarrow L^{d}$, 
$b \rightarrow \hat{b}^{d}$, and $\xi(t) \rightarrow \hat{\xi}(t)^{d}$. The volume-based expression can be used for the system where distance is not well defined, which is usual in infinite dimensional systems. Equation (2) can be applied to various singularities by choosing a proper function form of $\xi(t)$. A similar idea is seen in the finite size scaling method proposed by Kim $[18,19]$ [it uses correlation length $\hat{\xi}(t)$ directly observed in advance]. If we assume $\xi$ is a power function as $t^{-1 / y_{t}}$, Eq. (2) leads to

$$
g\left(t, h, N^{-1}\right)=b g\left(t b^{y_{t}}, h b^{y_{h}}, N^{-1} b\right),
$$

which is essentially the same as Eq. (1). On the other hand, by assuming an exponential function as $\xi(t)=$ $\exp \left[\left(t / t_{0}\right)^{-x_{t}}\right]$, we obtain

$$
g\left(t, h, N^{-1}\right)=b^{-1} \hat{g}\left(e^{-\left(t / t_{0}\right)^{-x_{t}}} b, h b^{y_{h}}, N^{-1} b\right) .
$$

Note that the present formula is applicable to normal phases, i.e., ordered or disordered phases, but not to critical phases where the correlation volume diverges.

We can calculate various physical quantities by differentiating the above free energy. Hereafter, we focus on the case of Eq. (4) for the ES in the ordered phase. The order parameter is given by

$$
\begin{aligned}
m\left(t, h, N^{-1}\right) & =g_{h}\left(t, h, N^{-1}\right) \equiv \partial g\left(t, h, N^{-1}\right) / \partial h \\
& =b^{-\left(1-y_{h}\right)} \hat{g}_{h}\left(e^{-\left(t / t_{0}\right)^{-x_{t}}} b, h b^{y_{h}}, N^{-1} b\right) .
\end{aligned}
$$

First, we consider the thermodynamic limit, $N=\infty$. By setting $b=e^{\left(t / t_{0}\right)^{-x_{t}}}$, we have

$$
m=e^{-\left(1-y_{h}\right)\left(t / t_{0}\right)^{-x_{t}}} \hat{g}_{h}\left(1, h e^{y_{h}\left(t / t_{0}\right)^{-x_{t}}}, 0\right) .
$$

Since $m$ is independent of $h$ for $h \rightarrow 0$ and independent of $t$ for $t \rightarrow 0$, the scaling function $g_{h}(1, x, 0)$ should have asymptotic forms as

$$
\hat{g}_{h}(1, x, 0)=\left\{\begin{array}{ll}
\text { const } & \text { for } x \ll 1 \\
x^{y_{h}^{-1}-1} & \text { for } x \gg 1
\end{array},\right.
$$

to reproduce

$$
m \propto\left\{\begin{array}{ll}
e^{-\left(1-y_{h}\right)\left(t / t_{0}\right)^{-x_{t}}} & \text { for } h e^{y_{h}\left(t / t_{0}\right)^{-x_{t}}} \ll 1 \\
h^{y_{h}^{-1}-1} & \text { for } h e^{y_{h}\left(t / t_{0}\right)^{-x_{t}}} \gg 1
\end{array} .\right.
$$

At $t=0$, it reads as $m \propto h^{1 / \delta}$ with $1 / \delta=y_{h}^{-1}-1$.

Second, we consider the case of $h=0$ with $N$ finite, where

$$
m=e^{-\left(1-y_{h}\right)\left(t / t_{0}\right)^{-x_{t}}} \hat{g}_{h}\left(1,0, N^{-1} e^{\left(t / t_{0}\right)^{-x_{t}}}\right) .
$$

The scaling function should be

$$
\hat{g}_{h}(1,0, x)=\left\{\begin{array}{ll}
\text { const } & \text { for } x \ll 1 \\
x^{1-y_{h}} & \text { for } x \gg 1
\end{array},\right.
$$

in a similar manner as Eq. (7) to reproduce

$$
m \propto\left\{\begin{array}{ll}
e^{-\left(1-y_{h}\right)\left(t / t_{0}\right)^{-x_{t}}} & \text { for } N^{-1} e^{\left(t / t_{0}\right)^{-x_{t}}} \ll 1 \\
N^{-\left(1-y_{h}\right)} & \text { for } N^{-1} e^{\left(t / t_{0}\right)^{-x_{t}}} \gg 1
\end{array} .\right.
$$

Similarly, susceptibility behaves as

$$
\chi \propto\left\{\begin{array}{ll}
e^{\left(2 y_{h}-1\right)\left(t / t_{0}\right)^{-x_{t}}} & \text { for } N^{-1} e^{\left(t / t_{0}\right)^{-x_{t}}} \ll 1 \\
N^{2 y_{h}-1} & \text { for } N^{-1} e^{\left(t / t_{0}\right)^{-x_{t}}} \gg 1
\end{array} .\right.
$$

The $m$-th derivative of the free energy with $h$, which is proportional to $\xi(t)^{\left(m y_{h}-1\right)}$, diverges at $t=0$ for $m>y_{h}^{-1}$. On the other hand, the derivative with $t$, such as specific heat, never diverges. Free energy for $h=0$ and $N=\infty$ is proportional to $\xi(t)^{-1}$, and the dominant term of its $m$-th derivative, $t^{-m\left(x_{t}+1\right)} \xi(t)^{-1}$, goes to zero for $t \rightarrow 0$.

Finally, we consider the case of $t=0$. By setting $b=N$ in Eq. (5), we have

$$
m=N^{-\left(1-y_{h}\right)} \hat{g}_{h}\left(0, h N^{y_{h}}, 1\right) .
$$

The scaling function should be

$$
\hat{g}_{h}(0, x, 1)=\left\{\begin{array}{ll}
x & \text { for } x \ll 1 \\
x^{y_{h}^{-1}-1} & \text { for } x \gg 1
\end{array},\right.
$$

to reproduce

$$
m \propto\left\{\begin{array}{ll}
h N^{2 y_{h}-1} & \text { for } h N^{y_{h}} \ll 1 \\
h^{y_{h}^{-1}-1} & \text { for } h N^{y_{h}} \gg 1
\end{array},\right.
$$

where we assumed linear susceptibility for a finite size system. This form is the same as that of the conventional PLS.

Next, we examine the validity of the present scaling ansatz by considering a hierarchical small-world network that is constructed in a recursive manner as shown in Fig. 1. The graph with $n$ generations has $N=2^{n-1}$ nodes and $3 \times 2^{n-1}-2$ edges. The degree distribution function is exponentially decaying as $P_{k} \propto 2^{-k / 2}$. We note the edges represented by the vertical lines in Fig. 1 as backbone edges (BBEs) and arcs as shortcut edges (SCEs).

The energy function of the $q$-state Potts model on the network under magnetic field $H$ is

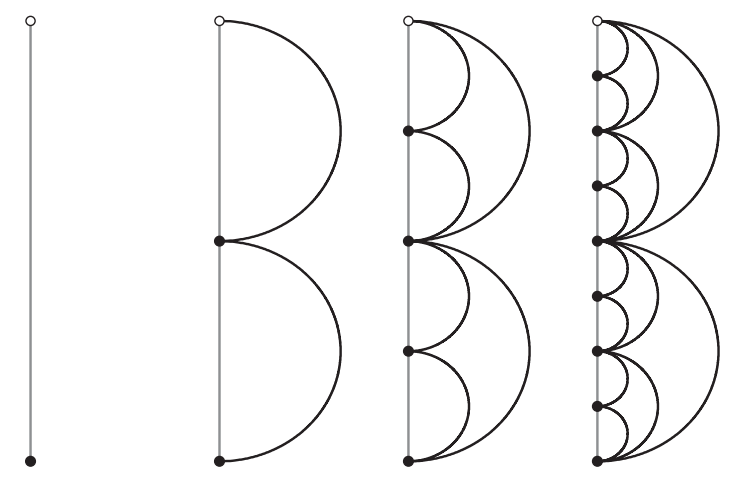

FIG. 1. Recursive construction of a shortcut network. The number of generation, $n$, equals $1,2,3$, and 4 from left to right. The vertical lines and solid arcs indicate backbone edges and shortcut edges, respectively. A periodic boundary condition is imposed in the vertical direction. The graph with $n=1$ is a single node with a self-connecting edge. 


$$
\begin{aligned}
-E / k_{B} T= & \sum_{\langle i, j\rangle \in \mathrm{BBE}}\left[K+D \delta_{\sigma_{i} 0}\right] \delta_{\sigma_{i} \sigma_{j}}+\sum_{\langle i, j\rangle \in \mathrm{SCE}} J \delta_{\sigma_{i} \sigma_{j}} \\
& +\sum_{i} H \delta_{\sigma_{i} 0}
\end{aligned}
$$

where $\delta_{a b}$ is the Kronecker delta and $\sigma_{i}$ is a spin variable at $i$-th site taking one of the values, $0,1, \cdots, q-1$. The first summation is over BBEs and the second one is over SCEs. We consider only the case $D=0$, but $D$ becomes finite in the real space RG performed below. In the following, we consider the case with $q \geq 3$ [20].

We can calculate the partition function of this system in a stepwise manner, decimating the spins in the youngest generation, which is the inverse procedure to grow the graph. The partial sum of the partition function preserves the function form by replacing the parameters of the energy function as

$$
\begin{aligned}
C_{n+1} e^{\left(K_{n+1}+D_{n+1} \delta_{\sigma_{i} 0}\right) \delta_{\sigma_{i} \sigma_{j}}+\left(H_{n+1} / 2\right)\left(\delta_{\sigma_{i} 0}+\delta_{\sigma_{j} 0}\right)} & \\
= & C_{n}^{2} e^{\left(H_{n} / 2\right)\left(\delta_{\sigma_{i} 0}+\delta_{\sigma_{j} 0}\right)} \\
& \times \sum_{\sigma_{k}=1}^{q} e^{\left(K_{n}+D_{n} \delta_{\sigma_{k} 0}+J\right)\left(\delta_{\sigma_{k} \sigma_{i}}+\delta_{\sigma_{k} \sigma_{j}}\right)+H_{n} \delta_{\sigma_{k} 0},},
\end{aligned}
$$

where $\sigma_{i}$ and $\sigma_{j}$ are nearest spins that are older than $\sigma_{k}$ by one generation. This gives us the following recursion relations:

$$
\begin{gathered}
g_{n+1}=g_{n}+A_{n}^{(4)} / 2^{n+1}, \\
K_{n+1}=A_{n}^{(2)}-A_{n}^{(4)}, \\
H_{n+1}=H_{n}+2\left(A_{n}^{(3)}-A_{n}^{(4)}\right), \\
D_{n+1}=A_{n}^{(1)}-A_{n}^{(2)}-2\left(A_{n}^{(3)}-A_{n}^{(4)}\right),
\end{gathered}
$$

with $g_{0}=0, K_{0}=K, H_{0}=H$, and $D_{0}=0$. Here

$$
\begin{gathered}
e^{A_{n}^{(1)}} \equiv e^{2\left(K_{n}+D_{n}+J\right)+H_{n}}+q_{1}, \\
e^{A_{n}^{(2)}} \equiv e^{2\left(K_{n}+J\right)}+e^{H_{n}}+q_{2}, \\
e^{A_{n}^{(3)}} \equiv e^{K_{n}+D_{n}+H_{n}+J}+e^{K_{n}+J}+q_{2}, \\
e^{A_{n}^{(4)}} \equiv 2 e^{K_{n}+J}+e^{H_{n}}+q_{3},
\end{gathered}
$$

and $q_{m}=q-m$. The quantity $g_{n} \equiv 2^{-n} \ln C_{n}$ is regarded as the free energy per spin of the system with $n$ generations. Note that $J$ does not change in this procedure.

In the case of no magnetic field, $H=0$, both $H_{n}$ and $D_{n}$ remain zero and Eq. (19) is rewritten as

$$
k_{n+1}=\frac{j k_{n}\left(q_{2} j k_{n}+2\right)}{q_{1} j^{2} k_{n}^{2}+1},
$$

where we put $k_{n} \equiv e^{-K_{n}}$ and $j \equiv e^{-J}$. The fixed point (FP) is obtained from $k_{n+1}=k_{n}=k^{*}$ as

$$
k^{*} \equiv 0, \quad \frac{q_{2}}{2 q_{1}}\left[1 \pm \sqrt{1+\frac{4 q_{1}}{q_{2}^{2}} \frac{2 j-1}{j^{2}}}\right]
$$

Figure 2 shows the RG fixed point and the phase boundary in $k$ vs $j$ space for $q=8$. This system exhibits a phase transition from the ferromagnetic phase corresponding to the FPs with $K=\infty$ to the phase corresponding to the FPs with finite $K$ by increasing $j$ at fixed $k$. We call the latter "critical phase" in the sense that the RG flow goes to neither $K=0$ nor $K=\infty$ but to nontrivial fixed points. The FP exhibits a saddle-node bifurcation (SNB) located

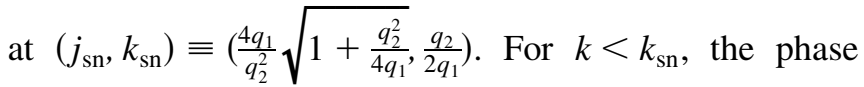
boundary is given by a line consisting of the unstable FPs, each of which leads to a PLS. On the other hand, a transition with ES occurs at $j=j_{\text {sn }}$ for $k \geq k_{\mathrm{sn}}$ and its singularity is governed by the SNB point. In the following, we consider the phase transition for $k=k_{\mathrm{sn}}$ in increasing $j$ (decreasing $J$ ).

First, we perform linear instability analysis around the SNB point. Equations (19)-(21) are approximated as

$$
\begin{gathered}
\left(\begin{array}{l}
K_{n+1} \\
D_{n+1} \\
H_{n+1}
\end{array}\right)=\mathrm{M}\left(\begin{array}{c}
K_{n} \\
D_{n} \\
H_{n}
\end{array}\right), \\
\mathrm{M} \equiv\left(\begin{array}{ccc}
1 & 0 & y\left(P_{1} y-P_{2}\right) \\
0 & 2\left(P_{1}-P_{2}\right) & (1-y)\left[P_{1}(1+y)-2 P_{2}\right] \\
0 & 2 P_{2} & 2 P_{2}(1-y)+1
\end{array}\right),
\end{gathered}
$$

with $P_{3} \equiv P_{1}-P_{2} y-1 / 2$. The largest eigenvalue is $\lambda_{+}$, and thus $H_{n}$ grows as $e^{n y_{h}}$ with $y_{h}=\ln \lambda_{+}$.

For $H=0$, only $\lambda=1$ is the relevant eigenvalue, which means that the instability is marginal as expected at the SNB point. Equation (26) is rewritten as

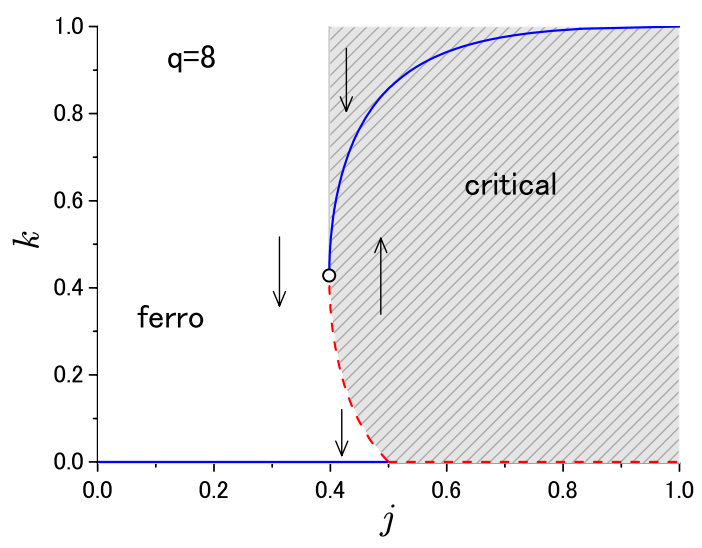

FIG. 2 (color online). Phase diagram for $q=8$. The shaded region indicates the critical phase. The (blue) solid line and the (red) dashed line denote the stable and unstable fixed lines, respectively. The circle symbol denotes the SNB point, $\left(j_{\mathrm{sn}}, k_{\mathrm{sn}}\right)$. 

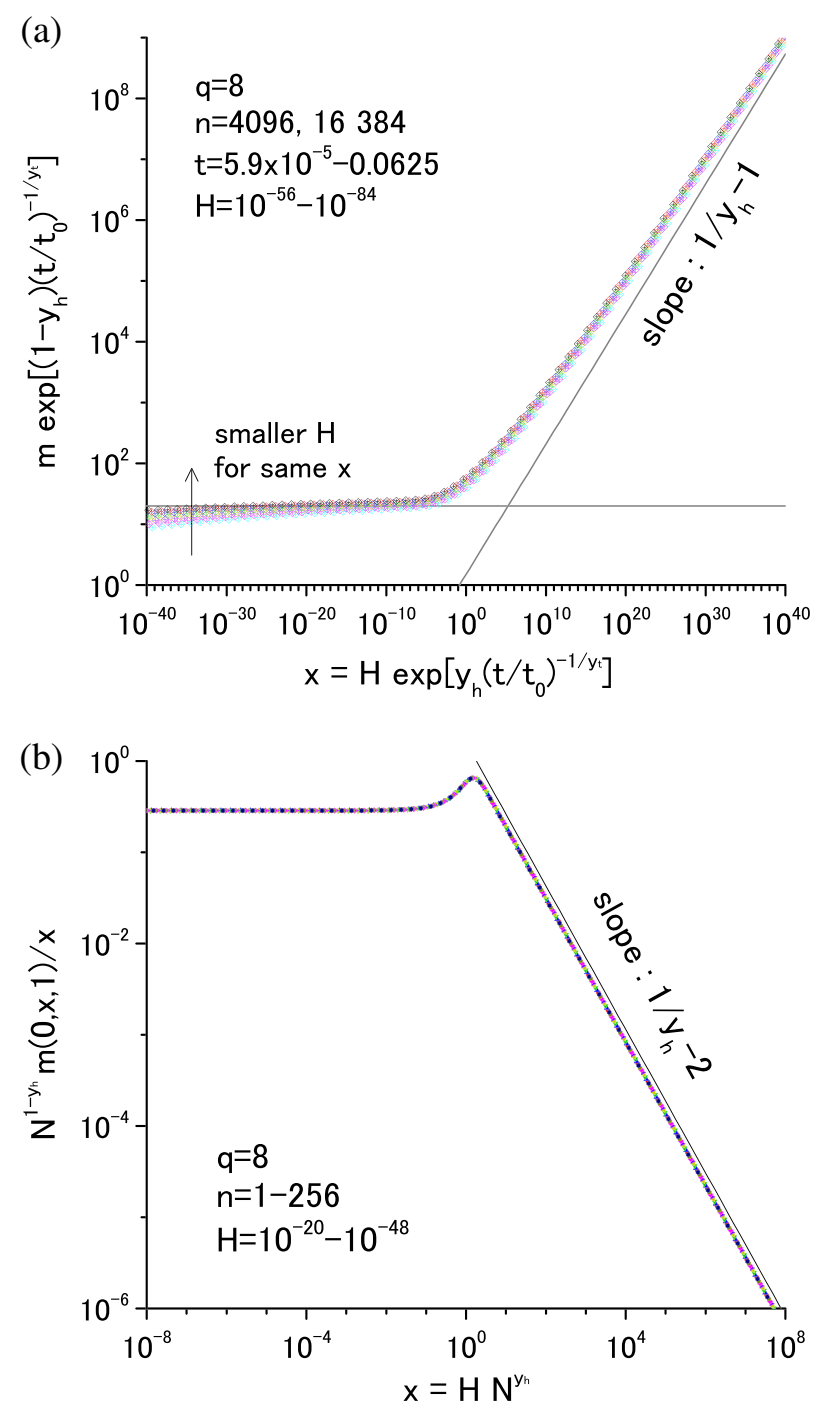

FIG. 3 (color online). Scaling plot of the order parameter for $1 / N \approx 0$ (a) and for $t=0$ (b). In the latter case, we show the data for $n=4096$ and 16384 to confirm the convergence to the large size limit.

$$
s_{n+1}-s_{n}=A\left(s_{n}^{2}+B t\right)
$$

in the lowest order of $s_{n}$ and $t$, where $s_{n}=k_{\mathrm{sn}}-k_{n}, t=$ $j_{\mathrm{sn}}-j, A \equiv \frac{q_{1} j_{\mathrm{sn}}^{2} k_{\mathrm{sn}}}{q_{1} j_{\mathrm{sn}}^{2} k_{\mathrm{sn}}^{2}+1}$, and $B=\frac{2\left(1-j_{\mathrm{sn}}\right)}{q_{1} j_{\mathrm{sn}}^{3}}$.

Equation (30) can be approximated to the so-called Kosterlitz equation [4], $d s / d n=A\left[s^{2}+B t\right]$, which is solved with $s_{n}(t)=\sqrt{B t} \tan [A n \sqrt{B t}]$ for the initial condition $s_{0}=0$. In this expression [valid until $s_{n}$ becomes $O(1)], s_{n}$ divergently grows as

$$
s_{n}(t)=1 / A\left(n_{0}(t)-n\right) \quad \text { with } \quad n_{0}(t) \equiv \pi / 2 A \sqrt{B t} .
$$

Now let us consider the scaling behavior of the present model. From the aforementioned analysis, the free energy behaves as

$$
g\left(s=0, t, H, N^{-1}\right)=2^{-n} g\left(n_{0}(t)-n, t, H e^{y_{h} n}, N^{-1} 2^{n}\right) .
$$

When we assume that the second argument $t$ does not yield any singularity, we obtain Eq. (4) by considering that

$$
\begin{aligned}
& b=2^{n}, \quad y_{h}=\ln \lambda_{+}, \\
& x_{t}=\frac{1}{2} \quad \text { and } \quad t_{0}=\frac{1}{B}\left(\frac{\pi \ln 2}{2 A}\right)^{2},
\end{aligned}
$$

where we use $2^{n_{0}(t)-n}=\xi(t) b^{-1}$. The value $x_{t}=1 / 2$ must have certain universality, because it is directly derived from the simplest nonlinear RG equation, $d s / d n \propto s^{2}+$ $B t$. This equation is a consequence of the SNB at the edge of the stable fixed line. We consider that this structure of the RG flow is the essence of the ES. In fact, it has been found in some systems [5,15-17].

Finally we confirm the scaling ansatz by calculating the order parameter, $m_{n}=\left(\tilde{m}_{n}-q^{-1}\right) /\left(1-q^{-1}\right)$, where $\tilde{m}_{n}=\partial\left[g_{n-1}+Z_{n-1}\right] / \partial H$ and $Z_{n}=e^{2\left(K_{n}+J+D_{n}+H_{n}\right)}+$ $q_{1}\left[e^{2\left(K_{n}+J\right)}+2 e^{H_{n}}+q_{2}\right]$. For this aim, we additionally calculate the derivatives of $g_{n}, K_{n}, D_{n}$, and $H_{n}$ with respect to $H$, whose recursion equations for these quantities are obtained by differentiating Eqs. (18)-(21).

Similarly, we calculate the susceptibility by using the second derivatives.

Figure 3(a) shows the scaling plot at $N \rightarrow \infty$ corresponding to Eqs. (7) and (8). Although we found a little correction to scaling that tends to disappear for $H \rightarrow 0$, a good collapse of data is obtained without any fitting parameter. In Fig. 3(b), we can see excellent scaling behavior for $t=j-j_{\text {sn }}=0$ corresponding to Eqs. (14) and (15). In both cases, the asymptotic form agrees with the prediction.

In conclusion, we have proposed a new scaling theory for the ES in infinite dimensional systems, and clarified its validity by analyzing a simple model. We believe that the present scaling formulas can be applied to various other models, and will clarify the existence of the universal mechanism for them. We have already confirmed that the scaling law holds in the bond percolation model on the decorated (2,2)-flower [15,16] (not shown here) and random growing network [22]. The finite size scaling formula included in the present theory will be useful in analyzing real-world data or numerical simulations, in which we can treat only small generations, $n \sim \log N$.

*nogawa@serow.t.u-tokyo.ac.jp

[1] K. G. Wilson, Rev. Mod. Phys. 47, 773 (1975).

[2] V.L. Berezinskii, Zh. Eksp. Teor. Fiz. 61, 1144 (1972) [Sov. Phys. JETP 34, 610 (1972)].

[3] J. M. Kosterlitz and D. J. Thouless, J. Phys. C 6, 1181 (1973).

[4] J. M. Kosterlitz, J. Phys. C 7, 1046 (1974).

[5] M. Hinczewski and A. N. Berker, Phys. Rev. E 73, 066126 (2006).

[6] M.E. J. Newman, S. H. Strogatz, and D. J. Watts, Phys. Rev. E 64, 026118 (2001).

[7] H. Shima and Y. Sakaniwa, J. Stat. Mech. (2006) P08017. 
[8] D. J. Watts and S. H. Strogatz, Nature (London) 393, 440 (1998).

[9] S. N. Dorogovtsev, A. V. Goltsev, and J. F. F. Mendes, Rev. Mod. Phys. 80, 1275 (2008).

[10] P. L. Krapivsky and B. Derrida, Physica A (Amsterdam) 340, 714 (2004).

[11] M. Bauer, S. Coulomb, and S. N. Dorogovtsev, Phys. Rev. Lett. 94, 200602 (2005).

[12] B. Bollobas and O. Riordan, Random Struct. Algorithms 27, 1 (2005).

[13] O. Riordan, Comb. Probab. Comput. 14, 897 (2005).

[14] S. Boettcher, J. L. Cook, and R. M. Ziff, Phys. Rev. E 80, 041115 (2009).

[15] A. N. Berker, M. Hinczewski, and R. R. Netz, Phys. Rev. E 80, 041118 (2009).
[16] T. Hasegawa, M. Sato, and K. Nemoto, Phys. Rev. E 82, 046101 (2010).

[17] S. Boettcher and C. T. Brunson, Phys. Rev. E 83, 021103 (2011).

[18] J.-K. Kim, Phys. Rev. Lett. 70, 1735 (1993).

[19] J.-K. Kim, Europhys. Lett. 28, 211 (1994).

[20] The present model with $q=2$ exhibits a behavior qualitatively different from those with $q \geq 3$, which is characterized by a pitchfork bifurcation of fixed points and will be discussed elsewhere. The percolation model $(q=1)$ on a similar network investigated in Ref. [21] also shows a pitchfork bifurcation.

[21] S. Boettcher, V. Singh, and R. M. Ziff, Nature Commun. 3, 787 (2012).

[22] T. Hasegawa, T. Nogawa, and K. Nemoto, arXiv:1009.6009. 